\title{
Accuracy of echogenic periportal enlargement image in ultrasonographic exams and histopathology in differential diagnosis of biliary atresia
}

\author{
Mariza L. V. Roquete, ${ }^{1}$ Alexandre R. Ferreira, ${ }^{2}$ Eleonora D. T. Fagundes, ${ }^{3}$ \\ Lúcia P. F. Castro, ${ }^{4}$ Rogério A. P. Silva, ${ }^{5}$ Francisco J. Penna 6
}

\begin{abstract}
Objectives: To define the sensitivity, specificity and accuracy of the ultrasound triangular cord sign and hepatic histopathology, in isolation or in combination, for diagnostic differentiation between biliary atresia and intrahepatic cholestasis.

Methods: This was a retrospective study carried out between January 1990 and December 2004. Fifty-one cases of biliary atresia and 45 of intrahepatic cholestasis were analyzed. Histopathology was performed blind by a pathologist. The triangular cord sign was identified in ultrasound reports as the only diagnostic sign of biliary atresia. Sensitivity, specificity and accuracy were calculated for the triangular cord sign and histology both in isolation and in combination. The gold standard for diagnosis of biliary atresia was the appearance of the extrahepatic biliary tree via laparotomy.

Results: The triangular cord sign alone had sensitivity of $49 \%$, specificity of $100 \%$ and accuracy of $72.5 \%$. Histopathology compatible with extrahepatic biliary obstruction alone had $90.2 \%$ sensitivity, $84.6 \%$ specificity and $87.8 \%$ accuracy. The triangular cord sign and histopathology in isolation or combination resulted in sensitivity of $93.2 \%$, specificity of $85.7 \%$ and accuracy of $90.3 \%$.
\end{abstract}

Conclusions: Finding the triangular cord sign on ultrasound is an indication for laparotomy. If the triangular cord sign is negative, liver biopsy is indicated; if histopathology reveals signs of biliary atresia, explorative laparotomy is indicated. In cases where the triangular cord sign is absent and histopathology indicates neonatal hepatitis or other intrahepatic cholestasis, clinical treatment or observation are recommended in accordance with the diagnosis.

J Pediatr (Rio J). 2008;84(4):331-336: Neonatal cholestasis, biliary atresia, ultrasound, liver biopsy.

\section{Introduction}

Neonatal cholestasis syndrome is one of the greatest challenges to pediatric hepatology, due to the countless possible causes and the need for rapid diagnosis of treatable conditions. Neonatal cholestasis can be classified into two major subsets, according to intrahepatic or extrahepatic causes. The intrahepatic group includes countless clinical entities of a metabolic, toxic or infectious nature. In contrast, the extrahepatic group - obstructive and surgical - is limited to a small number of conditions: biliary atresia (BA), choledochal cyst, bile duct stenosis and bile duct microlithiasis. The apparent simplicity of this classification of the cholestasis may catch out clinicians faced with a diagnostic dilemma that demands rapid and precise resolution. ${ }^{1}$

1. Doutora. Professora adjunta, Departamento de Pediatria, Faculdade de Medicina, Universidade Federal de Minas Gerais (UFMG), Belo Horizonte, MG, Brazil. 2. Doutor. Professor adjunto, Departamento de Pediatria, Faculdade de Medicina, UFMG, Belo Horizonte, MG, Brazil.

3. Pediatra. Doutora.

4. Professora associada, Departamento de Anatomia Patológica, Faculdade de Medicina, UFMG, Belo Horizonte, MG, Brazil.

5. Mestre, UFMG, Belo Horizonte, MG, Brazil. Médico, Instituto Alfa de Gastroenterologia, Hospital das Clínicas, UFMG, Belo Horizonte, MG e Centro Especializado em Ultra-Sonografia (CEU), Belo Horizonte, MG, Brazil.

6. Professor titular, Departamento de Pediatria, Faculdade de Medicina, UFMG, Belo Horizonte, MG, Brazil.

No conflicts of interest declared concerning the publication of this article.

Suggested citation: Roquete ML, Ferreira AR, Fagundes ED, Castro LP, Silva RA, Penna FJ. Accuracy of echogenic periportal enlargement image in ultrasonographic exams and histopathology in differential diagnosis of biliary atresia. J Pediatr (Rio J). 2008;84(4):331-336.

Manuscript received Mar 25 2008, accepted for publication May 282008.

doi:10.2223/JPED.1811 
Biliary atresia is responsible for $45 \%$ of cholestatic diseases in children. Prognosis is determined by surgical correction, using the Kasai procedure (hepatoportoenterostomy), before 60 days of life. ${ }^{1}$

When investigating a newborn or infant with jaundice secondary to conjugated hyperbilirubinemia, abdominal ultrasound (US) is one of the tests with greatest diagnostic relevance. Although it is considered operator-dependent, it is accessible, low-cost, noninvasive and does not depend on liver function. ${ }^{1}$ The lack of specificity for BA diagnosis of classical ultrasound findings has been replaced by the prospect of improving diagnostic accuracy based on a new sign visible on US - the triangular cord sign - which was first described by Korean authors. ${ }^{2-5}$ The triangular cord is a fibrous mass of triangular or tubular shape that is located at the cranial portion of the bifurcation of the portal vein and which is the ultrasonographic manifestation of the fibrous tissue remnant in the region of the porta hepatis. ${ }^{2}$

Notwithstanding, with the exception of explorative laparotomy which is the gold standard, liver biopsy is considered the best diagnostic test for BA. If the specimen contains five to seven portal spaces, accuracy can reach $93 \%$ at centers of excellence with pediatric pathologists trained in hepatology. ${ }^{6}$

The objective of this study is to define the sensitivity, specificity and accuracy of the triangular cord sign (TCS) on ultrasound and hepatic histopathology, in isolation or in conjunction, for differential diagnosis between BA and the intrahepatic cholestasis.

\section{Methods}

\section{Patients}

This was a retrospective study carried out at the Pediatric Hepatology Department at the Hospital das Clínicas at the Universidade Federal de Minas Gerais (UFMG) in Brazil. The patient sample was made up of those infants aged less than 6 months who were referred to the department between January of 1990 and December of 2004 for investigation of the causes of neonatal cholestasis syndrome. Patients who had undergone US and/or liver biopsy were included in the study. Infants were excluded if they were more than 6 months old, had ultrasound diagnoses of choledochal cyst or bile duct microlithiasis, had incomplete data on their medical records or their workup had been performed at another institution.

The study sample therefore comprised 96 infants with neonatal cholestasis. Fifty-one infants had a diagnosis of BA confirmed by explorative laparotomy combined with intraoperative cholangiography where feasible.

Age at the time of ultrasound varied from 6 to 155 days (mean: 68.6 \pm 32.0 ; median: 65.5). Liver biopsies were taken from patients aged from 5 to 155 days (mean: $79.4 \pm 30.6$ : median: 75.5).

\section{Abdominal ultrasound}

Ultrasound reports were checked for the presence or absence of TCS. The triangular cord sign, viewed on transverse and longitudinal scans following the portal vein, is seen on ultrasound as a structure of echogenic density, with thickness $\geq 3.0 \mathrm{~mm}$, and a triangular or tubular shape, located at the bifurcation of the portal vein affecting its first and second order branches. No other ultrasound findings were taken into account in this study.

Scans were performed by several different ultrasound specialists at the Radiology Department of the UFMG Hospital das Clínicas. A range of different systems were used, depending on date and circumstances, with 5.0 or $7.5 \mathrm{MHz}$ linear probes.

\section{Hepatic histopathology}

Liver biopsies were taken percutaneously in $52.7 \%$ of cases, using a Hepafix ${ }^{\circledR} 1.6$ needle; the remainder were surgical wedge biopsies. After being fixed in formol saline at $10 \%$, the liver specimen is processed using routine histological techniques, up to setting in paraffin; the paraffin block is sectioned with a microtome and, from each block, five slides are produced of 5.0 to $7.0 \mu \mathrm{m}$ thick histological sections, which are stained with hematoxylin and eosin (HE), reticulin, Perls (Prussian blue), Gomori's or Masson's trichrome and periodic acid-Schiff (PAS) with and without diastase. Slides with less than five portal spaces were excluded from the analysis.

Histopathological analysis of the liver biopsy slides was performed blind by a single experienced pathologist from the department.

\section{Definitive diagnosis}

Biliary atresia diagnoses were confirmed by explorative laparotomy by means of macroscopic identification of the atretic extrahepatic bile ducts and gallbladder and by operative cholangiography when it was feasible to inject a contrast medium into the pervious gallbladder. Laparotomy, with or without cholangiography, was therefore the gold standard for BA diagnosis, since it is the only procedure that can definitively confirm or rule out a diagnosis of BA. ${ }^{1,6,7}$ The surgical team comprises four pediatric surgeons.

In order to diagnose clinical or intrahepatic cholestasis, clinical follow-up was required, with remission of jaundice in up to 3 months of observation and assessment of clinical status plus the use of laboratory tests for certain diagnoses: idiopathic neonatal hepatitis, multifactorial cholestasis, Alagille syndrome, alpha-1-antitrypsin deficiency, tyrosinemia, galactosemia, and others.

\section{Statistics}

This is a retrospective study based on analysis of patient medical records.

Data analysis was performed using the public domain software program Epi-Info, version $6 .^{8}$ 


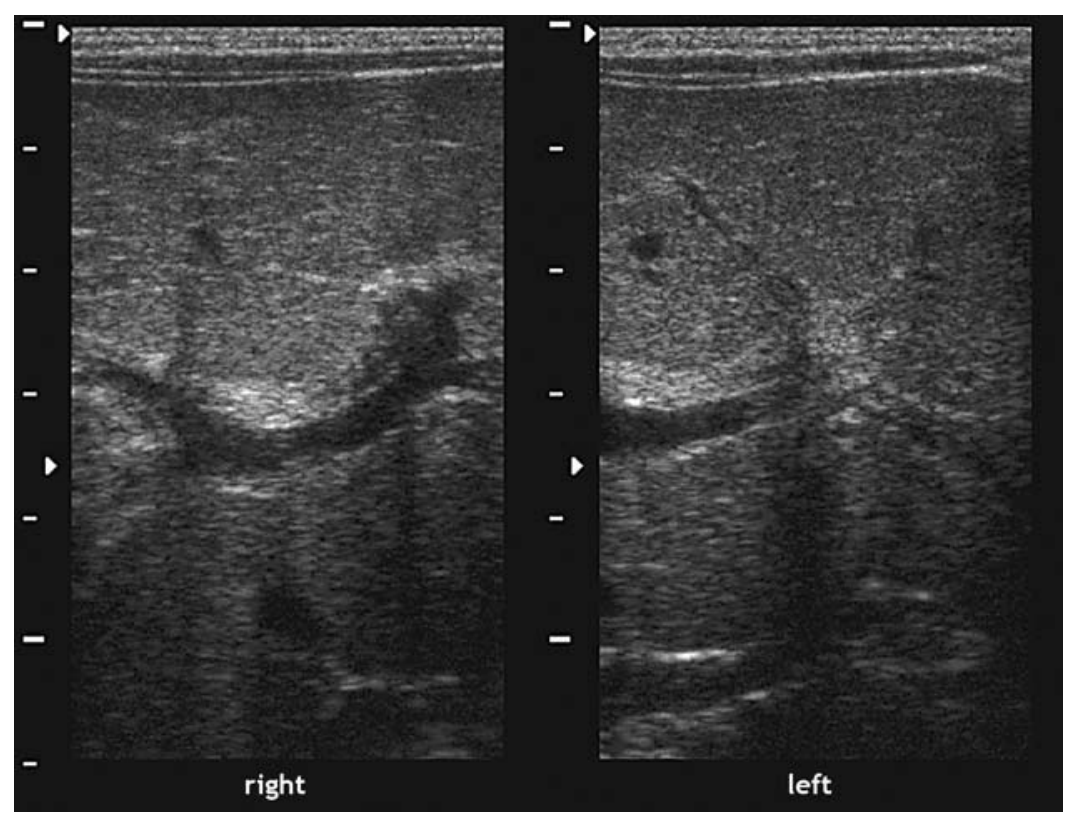

Figure 1 - Triangular cord sign on first and second order branches

Sensitivity, specificity and accuracy for the diagnosis of BA were calculated for the TCS and for histopathological findings of extrahepatic biliary obstruction in isolation. The same parameters were then calculated for two other cases: in the first case, both the TCS and histopathology compatible with extrahepatic biliary obstruction were obligatory for diagnosis; in the second, diagnosis could be made on the basis of one or the other being positive.

The study was approved by Research Ethics Committee at UFMG.

\section{Results}

When used as the only criterion for a diagnosis of BA, the TCS (Figure 1 ) had sensitivity of $49.0 \%$ (95\%CI 34.6-63.5) and specificity of $100 \%$ (95\%CI 89.6-100); accuracy was $72.5 \%$ (95\%CI 62.0-81.1). There were, therefore, no false-positives. Around $50 \%$ of cases of BA were, however, false-negative on US, being wrongly classified as intrahepatic cholestasis.

Histopathology findings compatible with extrahepatic obstruction of BA exhibited sensitivity of $90.2 \%$ ( $95 \% \mathrm{CI}$ 77.6-96.3), specificity of $84.6 \%$ (95\%CI 68.8-93.6) and accuracy of $87.8 \%$ ( $95 \%$ CI $78.8-93.4$ ) when used as the only criterion for diagnosis. Five of the 51 patients with BA were false-negative, while six out of 39 intrahepatic cholestasis patients who had histopathology compatible with BA, i.e., were false-positive.

When TCS and histopathology with extrahepatic obstruction were taken together for diagnosis of BA, sensitivity was $42.2 \%(95 \%$ CI $28.0-57.8)$ and specificity $100 \%$ (95\%CI
$86.3-100)$. Diagnostic accuracy was of the order of $65.8 \%$ (95\%CI 53.9-76.0).

When either of the two was accepted as sufficient for diagnosis, the results were as follows: sensitivity of $93.2 \%$ (95\% CI $80.3-98.2)$, specificity of $85.7 \%$ (95\% CI 66.4-95.3) and accuracy of $90.3 \%$ (95\%CI $80.4-95.7)$.

\section{Discussion}

This is a retrospective study and as such is subject to the limitations inherent to this type of design, such as the learning curve over time and changes related to improvements in equipment and/or techniques. Data on periportal thickening were obtained from a review of ultrasound reports. The scans were performed by several different ultrasound specialists since the patient load at the department is too great for all scans to be performed by a single professional. It was therefore decided to reflect the prevailing reality in including abdominal US scans that had been carried out by all of the medical professionals who do US scans at the Radiology Department of the UFMG Hospital das Clínicas. Despite this, it was possible to achieve $100 \%$ specificity, as shown by the pilot study by Pinto-Silva et al., ${ }^{9}$ where sensitivity was $62.5 \%$ using a single experienced examiner.

Table 1 is a synthesis of the experiences with ultrasound of a selection of authors and the results of this study, with emphasis on TCS for diagnosis of BA. ${ }^{4,10-14}$ Except in the study carried out in Japan, where scans were performed by two ultrasound specialists, all of the others used single examiners.

When this study is compared with those from Asia and Egypt, the sample from the UFMG Hospital das Clínicas is larger than the others as a result of the 15-year study period, 
Table 1 - Comparison between different authors' findings for sensitivity, specificity and accuracy of triangular cord sign for diagnosis of BA

\begin{tabular}{|c|c|c|c|c|c|}
\hline Authors & Country & $\mathbf{n}$ & Sensitivity (\%) & Specificity (\%) & Accuracy (\%) \\
\hline Park et al., $1997^{4}$ & Korea & 61 & 85.0 & 100.0 & 95.0 \\
\hline Kendrick et al., $2000^{10}$ & Singapore & 60 & 83.3 & 100.0 & 96.7 \\
\hline Kotb et al., $2001^{11}$ & Egypt & 60 & 100.0 & 100.0 & 100.0 \\
\hline Lee et al., $2003^{12}$ & Korea & 86 & 80.0 & 98.0 & 94.0 \\
\hline Visrutaratna et al., $2003^{13}$ & Thailand & 46 & 95.7 & 73.9 & 84.8 \\
\hline Kanegawa et al., $2003^{14}$ & Japan & 55 & 93.0 & 96.0 & 95.0 \\
\hline Roquete et al. & Brazil & 91 & 49.0 & 100.0 & 72.5 \\
\hline
\end{tabular}

$\mathrm{BA}=$ biliary atresia

which is a longer period than described by the other authors. The sensitivity of $49.0 \%$ found here is equivalent to one half to two-thirds of the values found by other studies, making the exam inadequate for screening at our department if used in isolation. This low sensitivity can be attributed to the heterogeneous nature of the ultrasound specialists' levels of experience at recognizing the TCS image. If, on one hand, the low sensitivity means that around half of BA cases were not diagnosed by US, the $100 \%$ specificity means that patients with intrahepatic cholestasis will not be unnecessarily subjected to explorative laparotomy. The TCS finding can speed up referral for laparotomy, making other diagnostic tests unnecessary, especially for patients who arrive at the department later. Training the ultrasound team involved with examining children could improve the sensitivity of this investigative technique at identifying TCS.

Twenty-five of the 49 BA cases that underwent ultrasound and where TCS was not found would have had their diagnosis of BA ruled out if persistent fecal acholia and hepatic histopathology had not also been available during workup. This demonstrates the need to include histopathology results in the differential diagnosis of infants with cholestasis.

The sensitivity, specificity and accuracy of hepatic histopathology observed here is listed in Table 2 together with other teams' experiences. ${ }^{15-21}$ The last two studies were performed in Brazil. This study was compared with the other two Brazilian studies in view of the institutional similarities. In common with our study, both the studies from the state of São Paulo describe patients treated at public university hospitals: Gastrocentro at the Medical Sciences Faculty of the Universidade Estadual de Campinas ${ }^{20}$ and the Instituto da Criança at the Universidade de São Paulo. ${ }^{21}$ The sample in our study was larger because it includes all cases of neonatal cholestasis over a 15-year period. The neonatal cholestasis samples in the Campinas and São Paulo studies were from periods of 4 years and 6 months and 6 years, respectively. ${ }^{20,21}$

From the total of 90 liver biopsies analyzed, based on the criterion of extrahepatic obstruction compatible with BA, 11 cases were wrongly diagnosed. There were six false-positives and five false-negatives. In view of the low sensitivity of US, it was combined with hepatic histopathology to improve diagnostic performance.

There was a discrete improvement over histopathology in isolation when either of the two diagnostic criteria was accepted for diagnosis. Sensitivity rose from 90.2 to $93.2 \%$, specificity from 84.6 to $85.7 \%$, and accuracy from 87.8 to $90.3 \%$.

The only definitive diagnostic test for BA remains, to date, explorative laparotomy, whether diagnosis is based on an atretic gallbladder seen macroscopically, replaced by fibrous remnants, or by intraoperative cholangiography. ${ }^{7,22}$ Nevertheless, less invasive preliminary tests capable of refining the indications for laparotomy are necessary to avoid unnecessarily subjecting patients with cholestasis due to intrahepatic causes to the procedure.

The low sensitivity of ultrasound means that combining it with liver biopsy results is indispensable to obtaining diagnostic confidence with relation to BA and, as a result, to safely indicating laparotomy for children with neonatal cholestasis. The merit of the TCS ultrasound sign lies in its $100 \%$ specificity, which can be of aid in speeding up referral for laparotomy, especially where infants arrive at the department aged more than 60 days. All of the cases that exhibited the sign were confirmed as BA. Due to the improved diagnostic performance offered by combining hepatic histopathology with the TCS, it is recommended that TCS and histopathology be combined for faster, more reliable, diagnoses. 
Table 2 - Comparison between different authors' results for sensitivity, specificity and accuracy of hepatic histopathology for diagnosing BA

\begin{tabular}{|c|c|c|c|c|}
\hline Source & $\mathbf{n}$ & Sensitivity (\%) & Specificity (\%) & Accuracy (\%) \\
\hline Manolaki et al., $1983^{15}$ & 86 & 90.0 & 82.5 & 86.5 \\
\hline Tolia et al., $1986^{16}$ & 24 & 95.6 & 90.0 & 93.9 \\
\hline Faweya et al., $1991^{17}$ & 27 & 83.3 & 100.0 & 92.6 \\
\hline Sanz \& Castilla, $1992^{18}$ & 78 & 89.0 & 95.5 & 93.0 \\
\hline Lai et al., $1994^{19}$ & 121 & 92.9 & 97.6 & 96.8 \\
\hline Hessel et al., $1994^{20}$ & 35 & 76.0 & 94.0 & 86.0 \\
\hline Zerbini et al., $1997^{21}$ & 74 & 100.0 & 75.9 & 90.5 \\
\hline Roquete et al. & 90 & 90.2 & 84.6 & 87.8 \\
\hline
\end{tabular}

$\mathrm{BA}=$ biliary atresia

The results of this study agree with the algorithm proposed by Kotb et al.: ${ }^{11}$ the TCS sign seen on ultrasound is enough to indicate laparotomy with cholangiography, if this is feasible. If the TCS is negative, liver biopsy is indicated; if histopathology shows signs of BA, explorative laparotomy is once more indicated. In cases where TCS is negative and histopathology indicates neonatal hepatitis or other intrahepatic cholestasis, clinical treatment or observation are recommended in accordance with the diagnosis.

\section{References}

1. Bernard O. Cholestatic childhood liver diseases. Acta Gastroenterol Belg. 1999;62:295-9.

2. Choi SO, Park WH, Lee HJ, Woo SK. 'Triangular cord': a sonographic finding applicable in the diagnosis of biliary atresia. J Pediatr Surg. 1996;31:363-6.

3. Choi SO, Park WH, Lee HJ. Ultrasonographic "triangular cord": the most definitive finding for noninvasive diagnosis of extrahepatic biliary atresia. Eur J Pediatr Surg. 1998;8:12-6.

4. Park WH, Choi SO, Lee HJ, Kim SP, Zeon SK, Lee SL. A new diagnostic approach to biliary atresia with emphasis on the ultrasonographic triangular cord sign: comparison of ultrasonography, hepatobiliary scintigraphy, and liver needle biopsy in the evaluation of infantile cholestasis. J Pediatr Surg. 1997;32:1555-9.

5. Park WH, Choi SO, Lee HJ. The ultrasonographic 'triangular cord' coupled with gallbladder images in the diagnosis prediction of biliary atresia from infantile intrahepatic cholestasis. J Pediatr Surg. 1999;34:1706-10.

6. Schreiber RA, Kleinman RE. Biliary atresia. J Pediatr Gastroenterol Nutr. 2002;35 Suppl 1:S11-6.

7. Middlesworth W, Altman RP. Biliary atresia. Curr Opin Pediatr. 1997;9:265-9.
8. Dean AG, Dean JA, Culombier D. Epi Info, version 6; a word processing, database, and statistics program for epidemiology on microcomputers. Atlanta, GA: Center for Disease Control and Prevention; 1994.

9. Pinto-Silva RA, Roquete ML, Ferreira AR, Penna FJ, Lobo BM, Silveira JL, et al. Espessamento ecogênico periportal: achado ultra-sonográfico sugestivo de $A B$ extra-hepática. Acta Radiol Paulista. 1998;1:49-53.

10. Tan Kendrick AP, Phua KB, Ooi BC, Subramaniam R, Tan CE, Goh AS. Making the diagnosis of biliary atresia using the triangular cord sign and gallbladder length. Pediatr Radiol. 2000; 30:69-73.

11. Kotb MA, Kotb A, Sheba MF, El Koofy NM, El-Karaksy HM, Abdel-Kahlik MK, et al. Evaluation of the triangular cord sign in the diagnosis of biliary atresia. Pediatrics. 2001;108:416-20.

12. Lee HJ, Lee SM, Park WH, Choi SO. Objective criteria of triangular cord sign in biliary atresia on US scans. Radiology. 2003;229:395-400.

13. Visrutaratna $P$, Wongsawasdi $L$, Lerttumnongtum $P$, Singhavejsakul J, Kattipattanapong V, Ukarapol N. Triangular cord sign and ultrasound features of the gall bladder in the infants with biliary atresia. Australas Radiol. 2003;47:252-6.

14. Kanegawa K, Akasaka Y, Kitamura E, Nishiyama S, Muraji T, Nishijima E, et al. Sonographic diagnosis of biliary atresia in pediatric patients using the "triangular cord" sign versus gallbladder length and contraction. AJR Am J Roentgenol. 2003; 181:1387-90.

15. Manolaki AG, Larcher VF, Mowat AP, Barrett JJ, Portmann B, Howard ER. The prelaparotomy diagnosis of extrahepatic biliary atresia. Arch Dis Child. 1983;58:591-4.

16. Tolia V, Dubois RS, Kagalwalla A, Fleming S, Dua V. Comparison of radionuclear scintigraphy and liver biopsy in the evaluation of neonatal cholestasis. J Pediatr Gastroenterol Nutr. 1986;5: 30-4.

17. Faweya AG, Akinyinka OO, Sodeinde O. Duodenal intubation and aspiration test: utility in the differential diagnosis of infantile cholestasis. J Pediatr Gastroenterol Nutr. 1991;13:290-2.

18. Sanz CR, Castilla EN. Papel de la biopsia hepática en el diagnóstico de la colestasis prolongada em lactentes. Rev Invest Clin. 1992;44:193-202. 
19. Lai MW, Chang MH, Hsu SC, Hsu HC, Su CT, Kao CL, et al. Differential diagnosis of extrahepatic biliary atresia from neonatal hepatitis: a prospective study. J Pediatr Gastroenterol Nutr. 1994; 18:121-7.

20. Hessel G, Yamada RM, Escanhoela CA, Bustorff-Silva JM, Toledo RJ. Valor da ultra-sonografia abdominal e da biopsia hepática percutânea no diagnóstico diferencial da colestase neonatal. Arq Gastroenterol. 1994;31:75-82.

21. Zerbini MC, Galluci SD, Maezono R, Ueno CM, Porta G, Maksoud JG, et al. Liver biopsy in neonatal cholestasis: a review on statistical grounds. Mod Pathol. 1997;10:793-9.
22. de Carvalho E, Ivantes CA, Bezerra JA. Extrahepatic biliary atresia: current concepts and future directions. J Pediatr (Rio J). 2007;83:105-20.

Correspondence:

Mariza L. V. Roquete

Rua Professor Norton Kaiserman, 82/102 - Anchieta

CEP 30310-570 - Belo Horizonte, MG - Brazil

E-mail: roquetemlv@uol.com.br 